\title{
Resolving the Atomic Structure of Sequential Infiltration Synthesis Derived Inorganic Clusters
}

Xiang He, ${ }^{\dagger}$ Ruben Z. Waldman, ${ }^{\dagger \perp}$ David J. Mandia," Nari Jeon, ${ }^{\dagger /}$ Nestor J. Zaluzec, ${ }^{\dagger}$ Olaf J. Borkiewicz, ${ }^{\S}$ Uta Ruett, ${ }^{\S}$ Seth B. Darling, ${ }^{\dagger \perp}$ Alex B. F. Martinson, ${ }^{\dagger / *}$ and David M. Tiede ${ }^{\dagger * *}$

'Advanced Materials for Energy-Water Systems (AMEWS) Energy Frontier Research Center, Chemical Sciences and Engineering Division, "Materials Science Division, \#Photon Sciences Division, and ${ }^{\S}$ X-ray Science Division, Argonne National Laboratory, Lemont, IL 60439, USA

${ }^{\perp}$ Pritzker School of Molecular Engineering, University of Chicago, Chicago, IL 60637, United States

Corresponding Authors:

*martinson@anl.gov

*tiede@anl.gov 

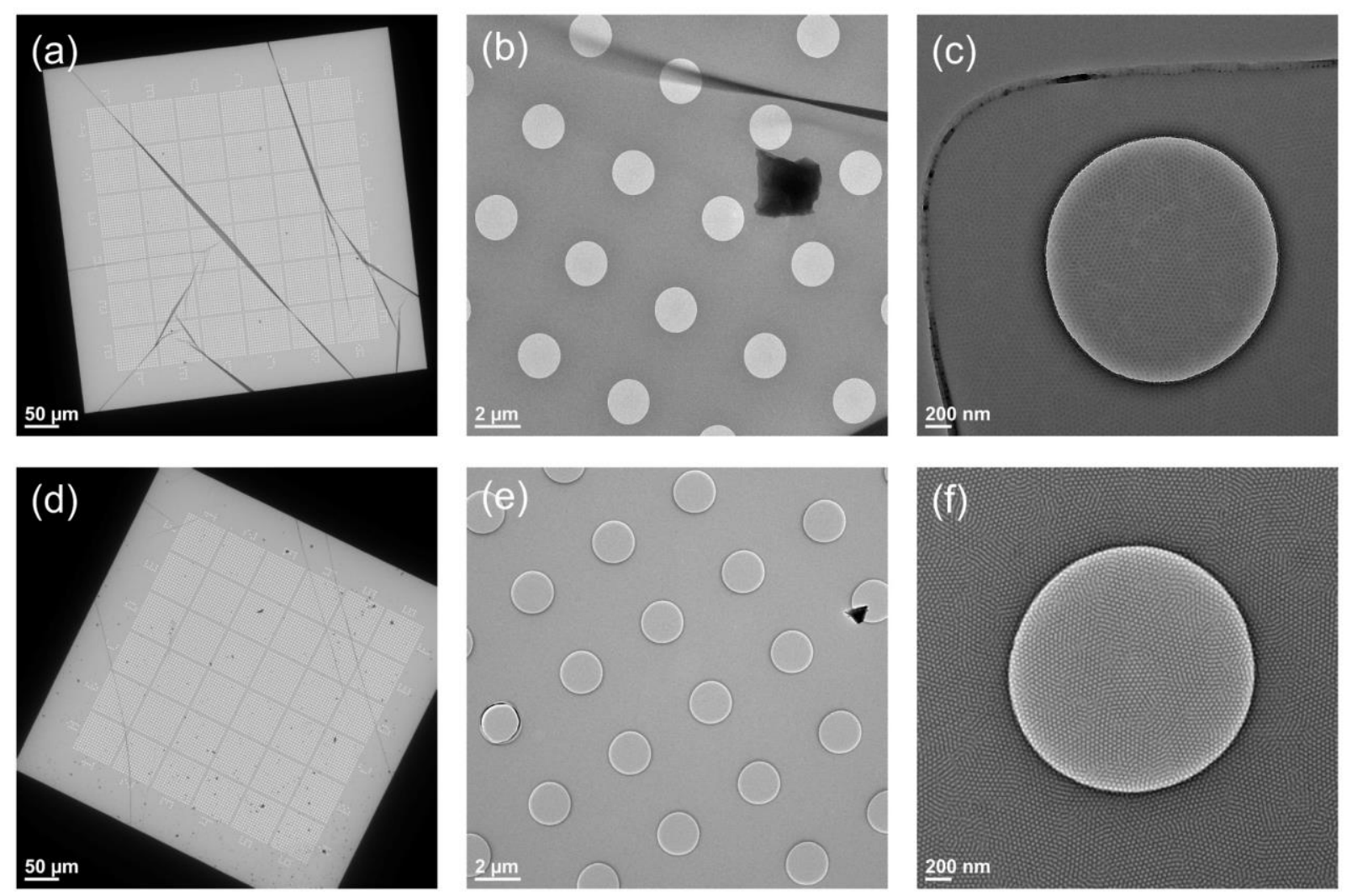

Figure S1. TEM images of block copolymer film covered TEM grid before (a, b, c) and after (c, d, e) three cycles of SIS processes.

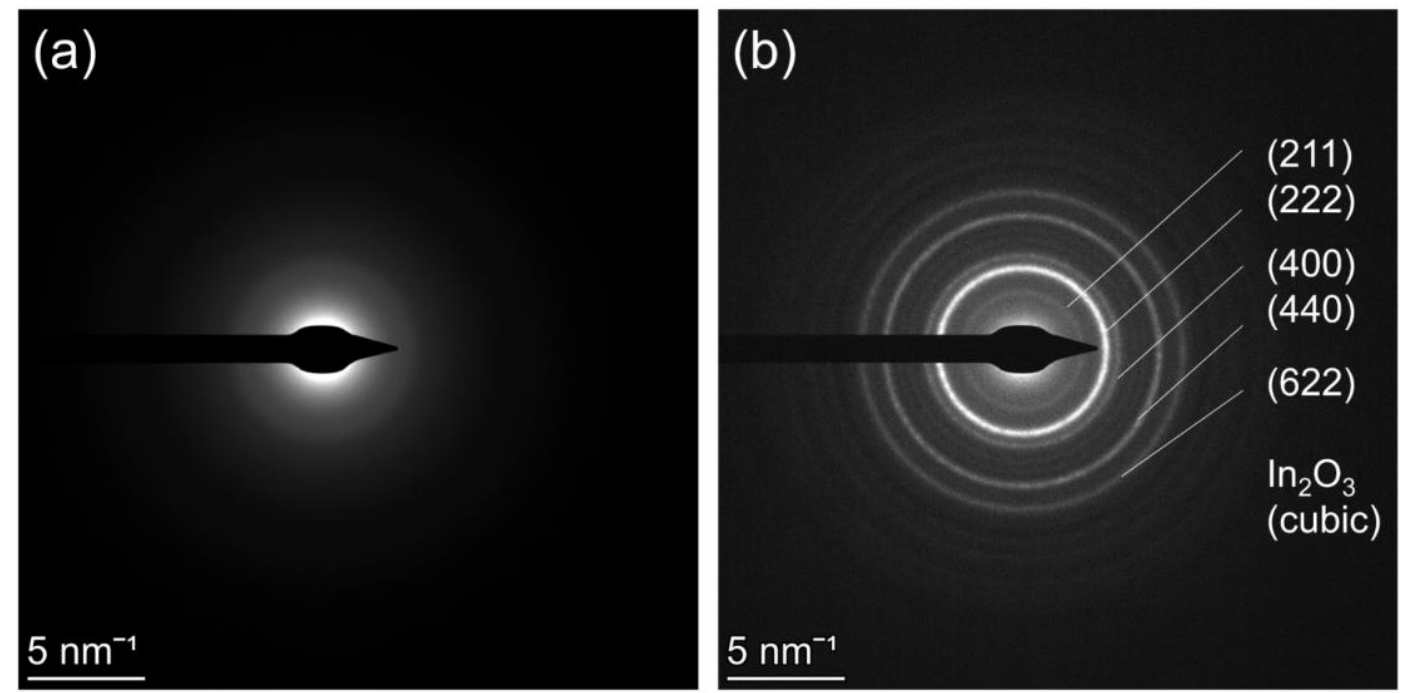

Figure S2. TEM diffraction data of (a) 3AG and (b) 3Ann obtained using block copolymer template. 

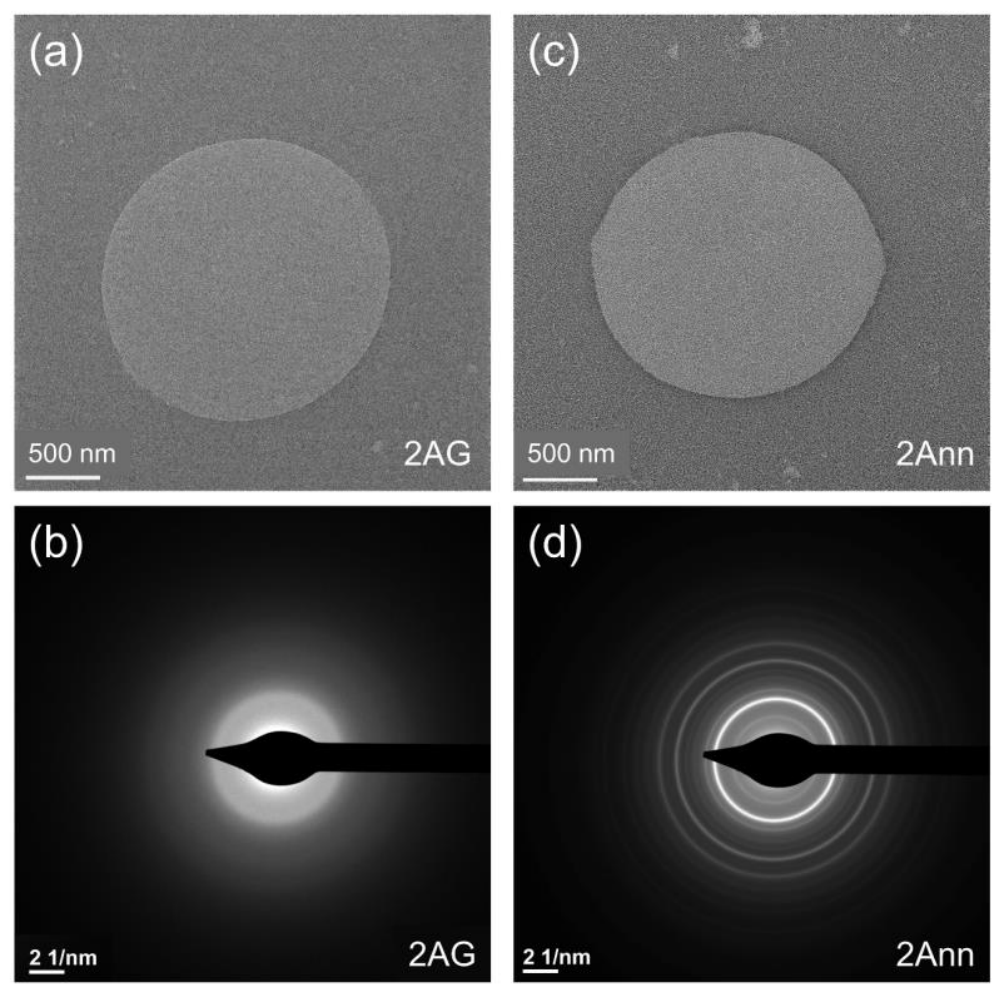

Figure S3. TEM images and diffraction data of (a, b) 2AG and (c, d) 2Ann prepared with PMMA on TEM grids.
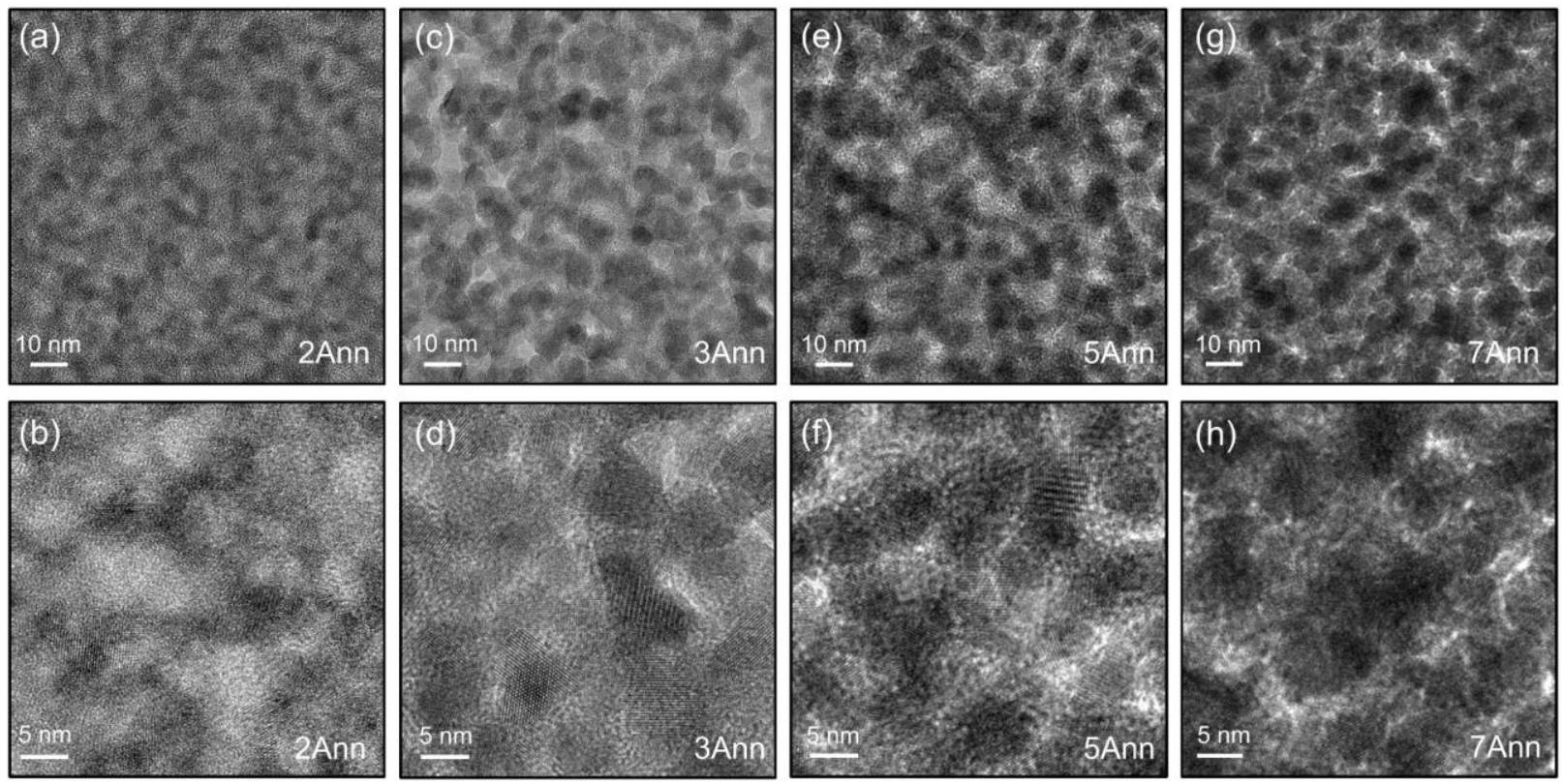

Figure S4. TEM images of annealed SIS samples prepared with homopolymer (i.e., PMMA): (a, b) 2 cycles, (c, d) 3 cycles, (e, f) 5 cycles, $(\mathrm{g}, \mathrm{h}) 7$ cycles. 


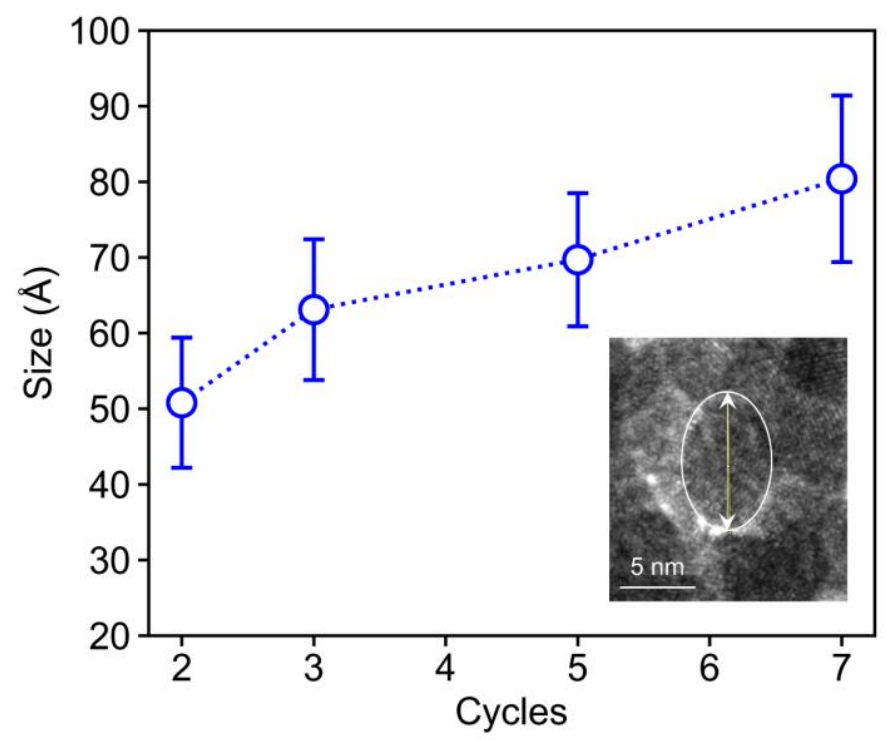

Figure S5. Variation of the maximal nanograin dimension in annealed PMMA-templated $\operatorname{In}_{2} \mathrm{O}_{3}$ samples determined by individual particle selection in TEM images. Inset: a representative nanograin identified for size measurement, where the arrow line indicates the major axis dimension used as the size of that nanograin. For each sample, at least 50 nanograins are measured to get the average size and standard deviation.

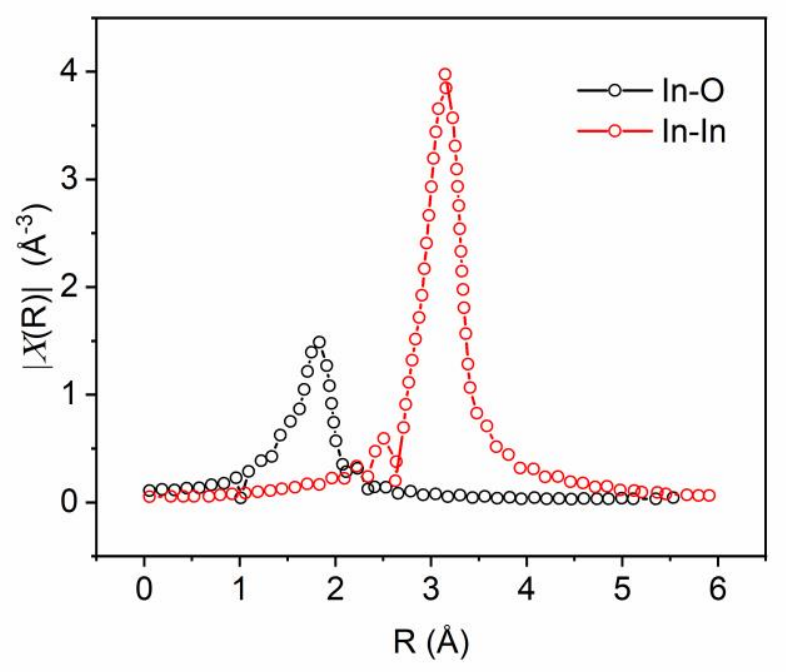

Figure S6. Theoretical FEFF calculation of the In-O and In-In single scattering pathways used in (generated from c- $\operatorname{In}_{2} \mathrm{O}_{3} \mathrm{CIF}$ (mp-22598) information) EXAFS fitting analysis of the $1^{\text {st }}$ and $2^{\text {nd }}$ coordination shells for In-O and In-In interactions (In absorber), respectively. (Note: In-O in its octahedral coordination has degeneracy of $\mathrm{N}=6$, and the tetrahedrally-coordinated, $\mathrm{N}=4, \mathrm{In}-\mathrm{O}$

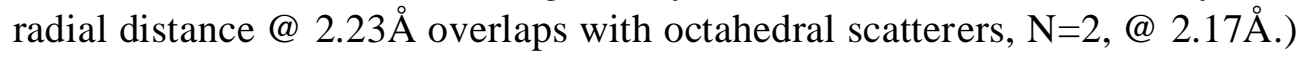



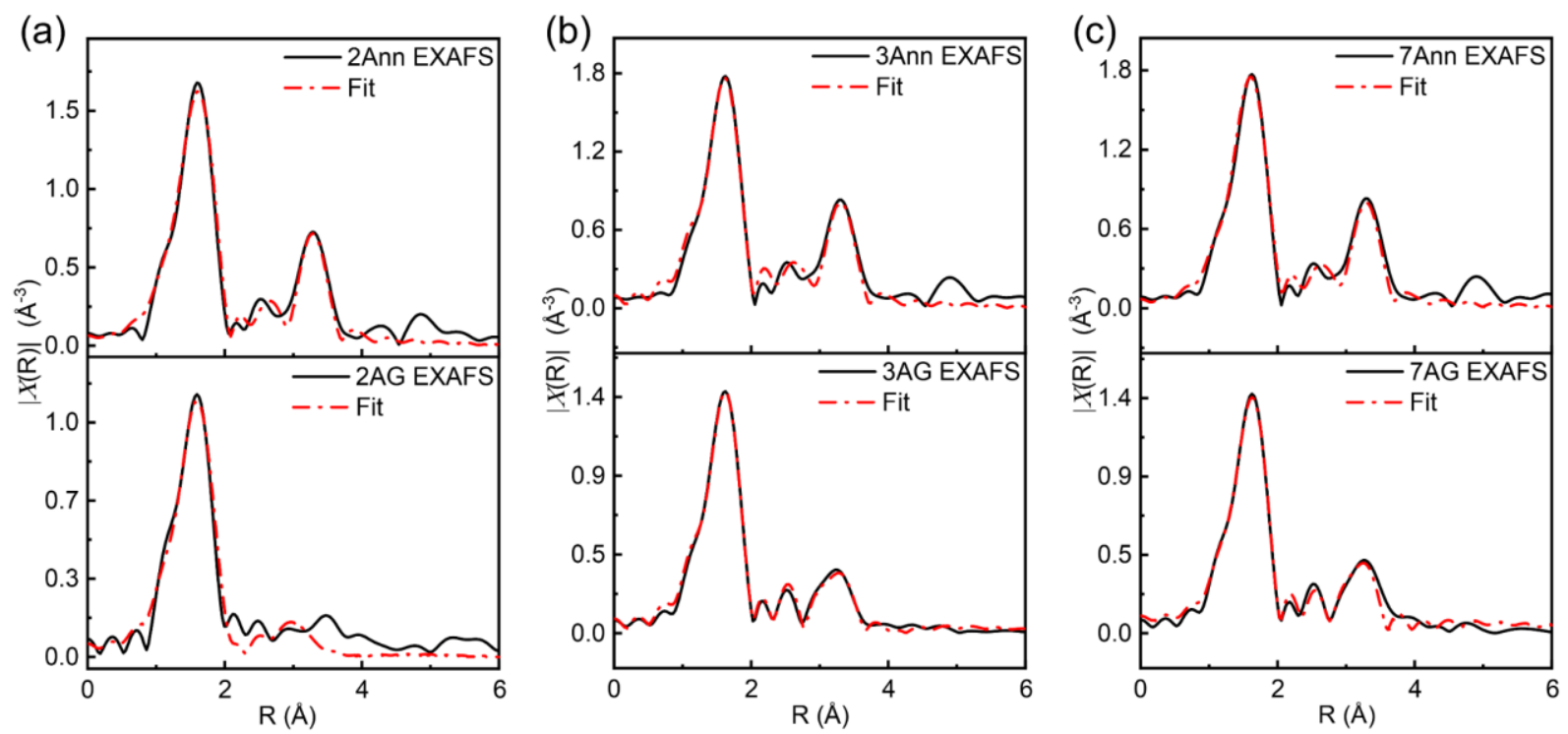

Figure S7. 2-shell, 2-single-scattering path (SSP) EXAFS fitting of the (a) 2AG (bottom) and 2Ann (top) samples, (b) 3AG (bottom) and 3Ann (top) samples (c) 7AG (bottom) and 7Ann (top) samples.

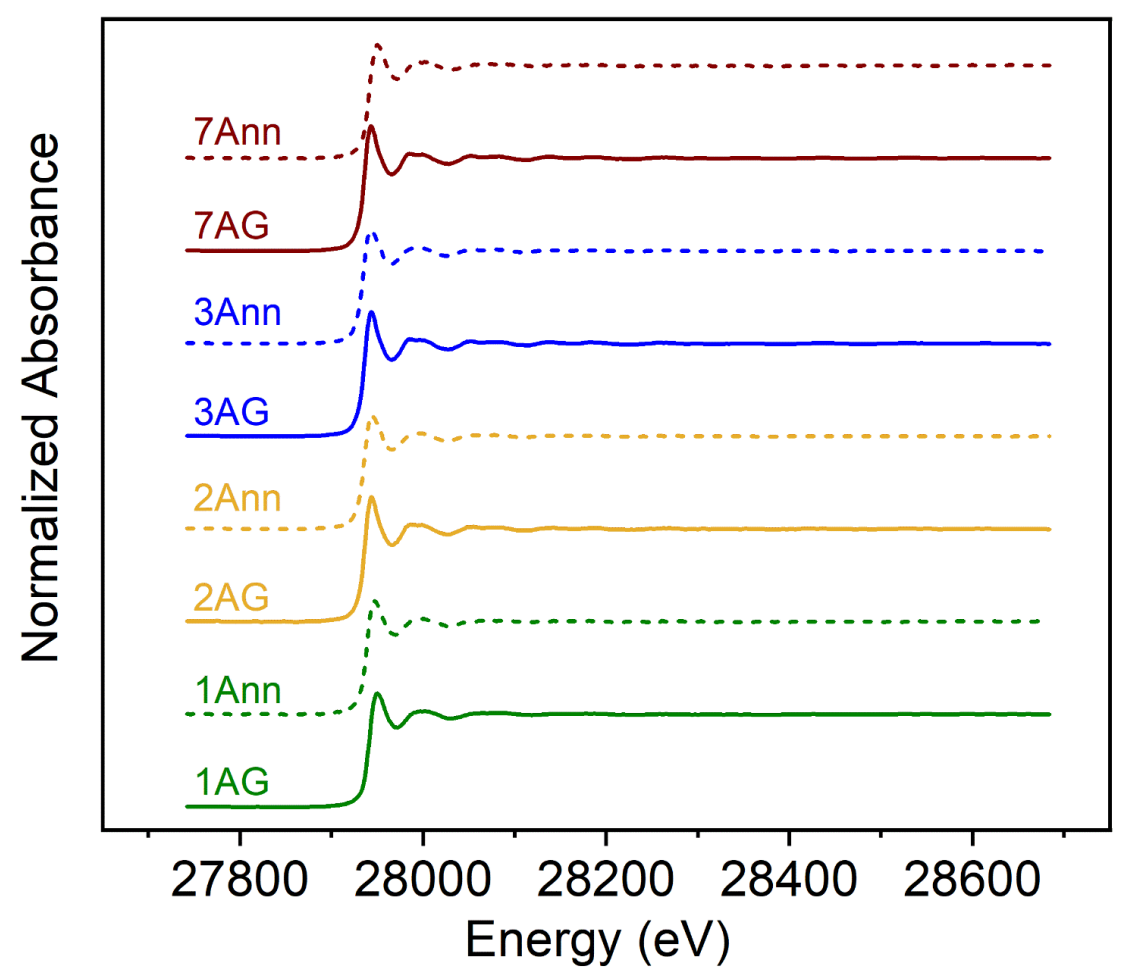

Figure S8. In K edge XANES spectra of SIS samples. 
(a)

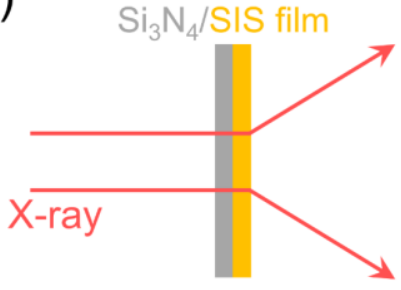

(b)

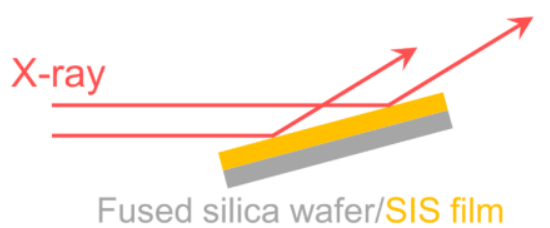

Grazing Incidence

(c)

Transmission

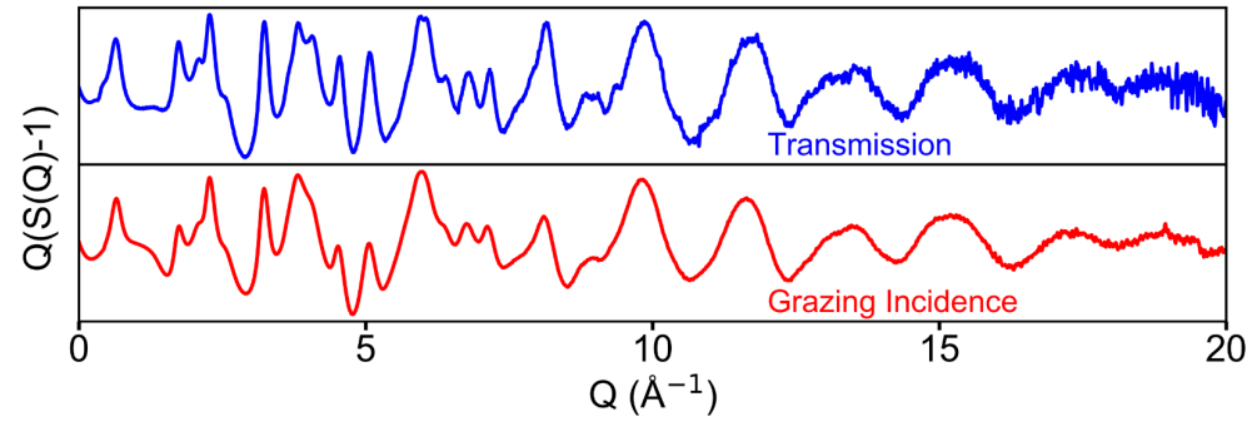

(d)

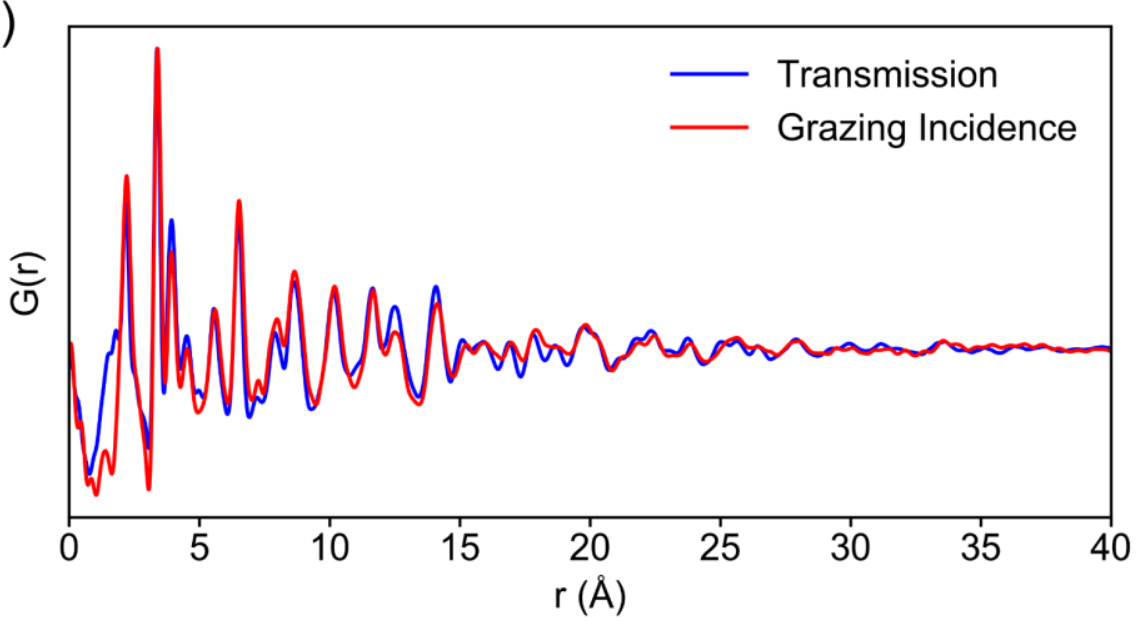

Figure S9. Collection of HEXS data using (a) transmission geometry and (b) grazing-incidence geometry with a focused beam of 5 um height. Comparison of (c) Reduced structure functions and (d) PDF patterns of 7AG measured from two different geometries. In comparison, the HEXS data obtained from both geometries offer similar signal-to-noise (Figure S9c) and PDF interrogation (Figure S9d). However, for the transmission mode, we needed to use $200 \mathrm{~nm} \mathrm{Si} 3 \mathrm{~N}_{4}$ substrates to minimize the background contribution and longer, $50 \mathrm{~min}$. total (5 sets of $10 \mathrm{~min}$. HEXS image acquisitions) for sample and background data acquisitions. In contrast, data collection using grazing-incidence geometry did not have special requirements for the substrate, here we used glass slides, and it is much more efficient than the transmission for data acquisition. Here we used 5 min. 
total acquisition time (sum of 300, 1-sec exposures), which also creates opportunities to monitor the in-situ growth of SIS thin films in the future. More details about GI measurement can be found below:

The grazing-incidence measurements have been carried out at beamline 11-ID-B at a photon energy of $87.6 \mathrm{keV}(0.143 \AA$ ) using a custom-made sample holder consisting of a $10-\mathrm{mm}$ pin, goniometer head and centering assembly mounted to Physik Instrumente H-840 hexapod. The specimen is secured to the pin through suction provided by a series of channels running along the height of the pin attached to a vacuum pump. These channels serve a second purpose as alignment pattern (cross) on the face of the pin, used during mounting of the specimen and standard materials (calibrants) to ensure consistent positioning of the samples with respect to the X-ray beam. The point of confusion of the hexapod, assigned to the center of the top surface of the sample (taking into account the height of the pin and the thickness of the specimen), is aligned to X-rays. The calibration of the measurement is carried out using NIST-traceable SRM 674b $\mathrm{CeO}_{2}$ material loaded into a 1-mm OD Kapton capillary mounted in the center plane of the pin perpendicular to the path of the X-rays using the aforementioned alignment pattern of the pin surface. This approach generates calibration parameters for the center plane of the pin, which remain valid for the measurements of other samples: the footprint of the beam during the measurements on the flat specimen, if aligned correctly, remains centered on that plane. Data has been collected on a PerkinElmer 1621 a-Si detector using 300, 1-second exposures summed into one image for a total collection time of 5 minutes per data point. The length of the exposure has been optimized to yield satisfactory signal quality without overexposing the detector. Data has been acquired using the QXRD program; dark current and flat-field corrections have been applied within the same software. GSAS-II package has been used for measurement calibration and data reduction and integration. 

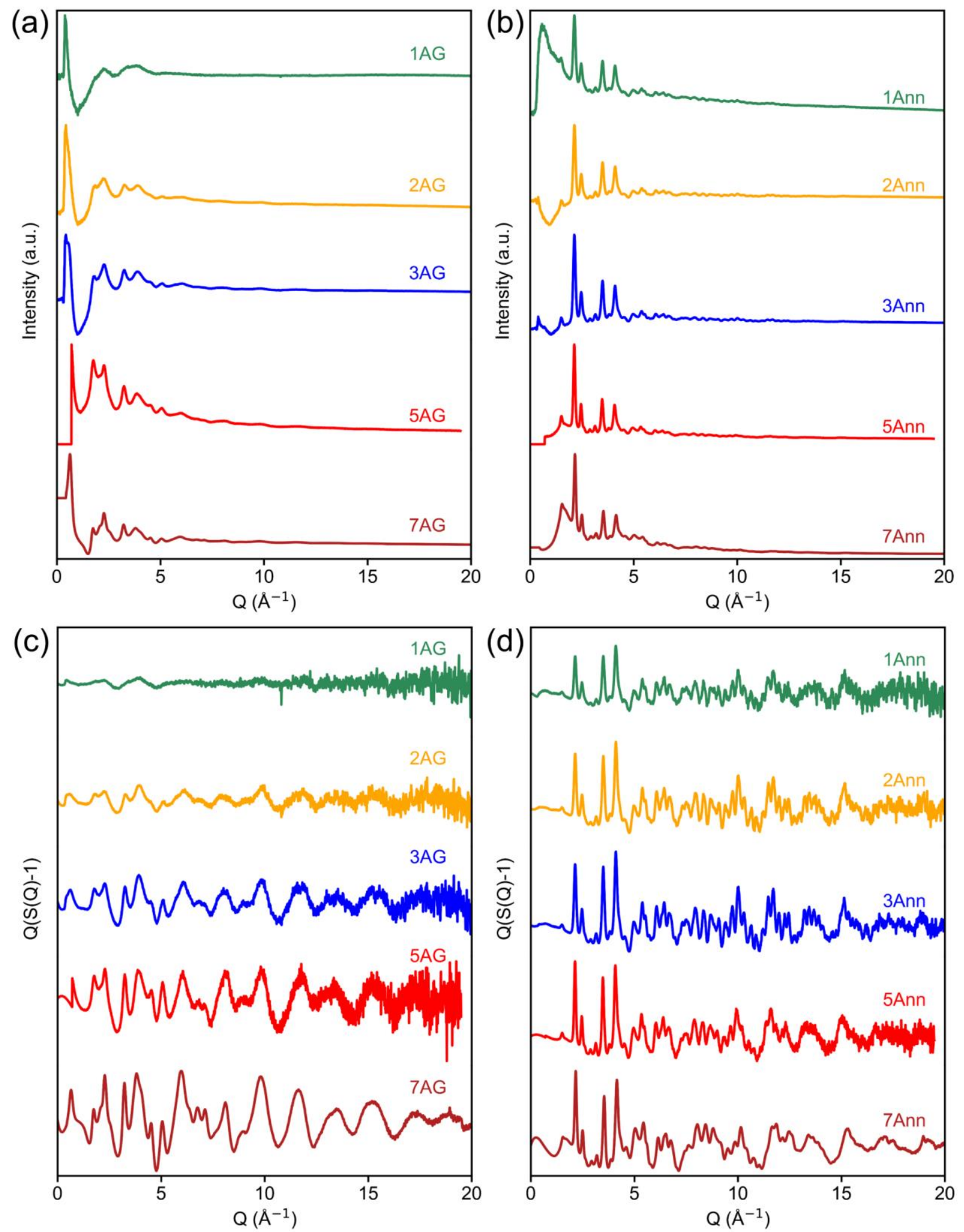

Figure S10. Full sets of data: (a, b) synchrotron XRD patterns, (c, d) reduced structure functions. 


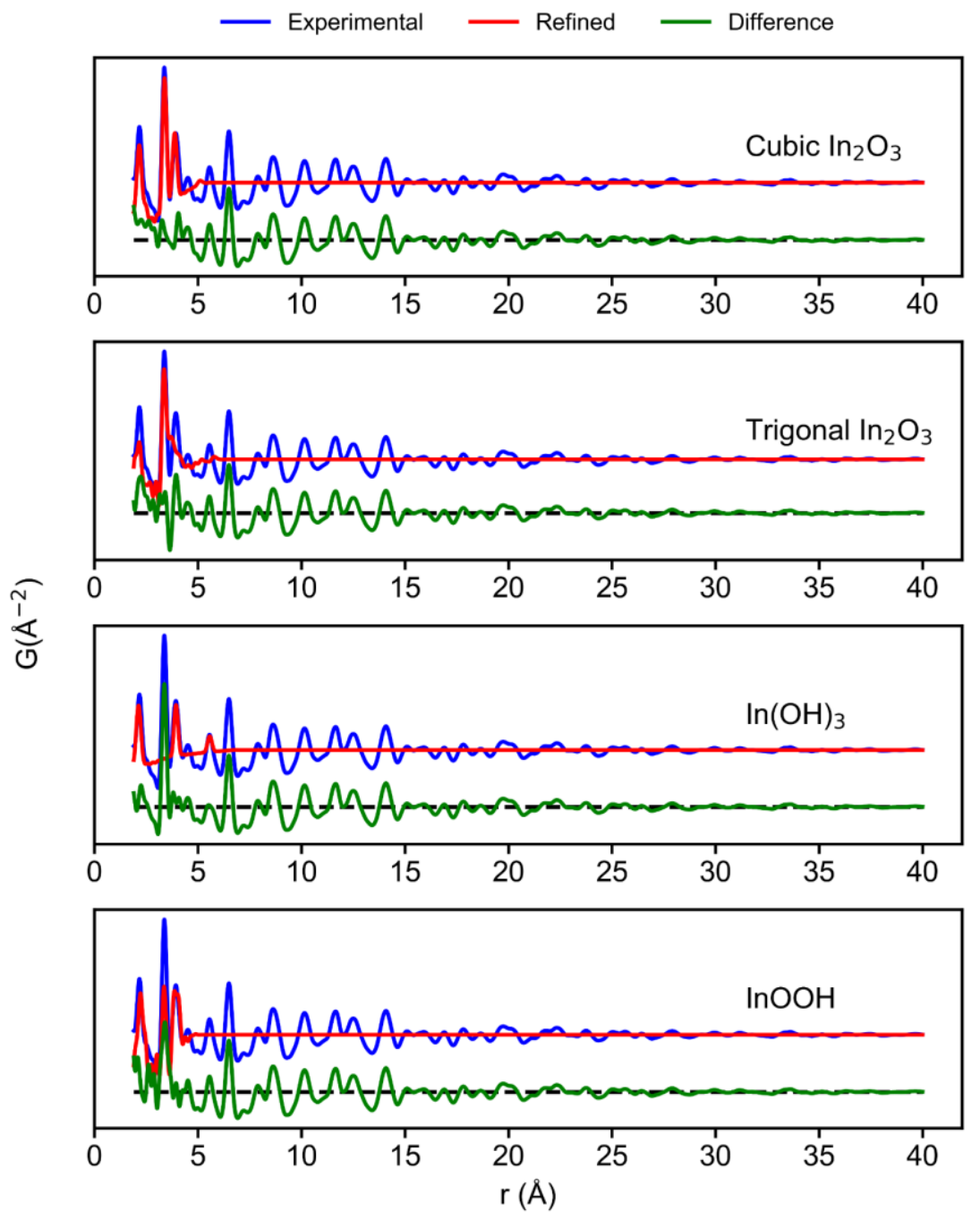

Figure S11. Refinement of the PDF of 7AG using four standard crystalline structures using Diffpy-CMI ${ }^{1}$ and details were described in the Experimental Procedure section of the main text. 
(a)

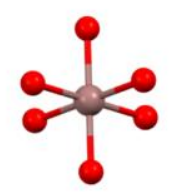

(1)
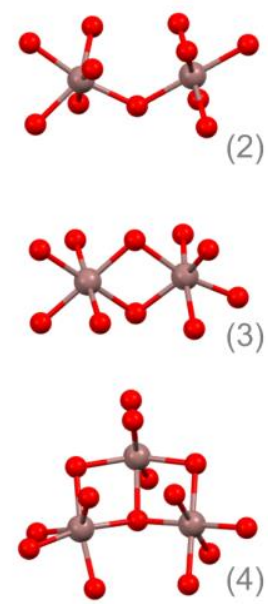

(b)

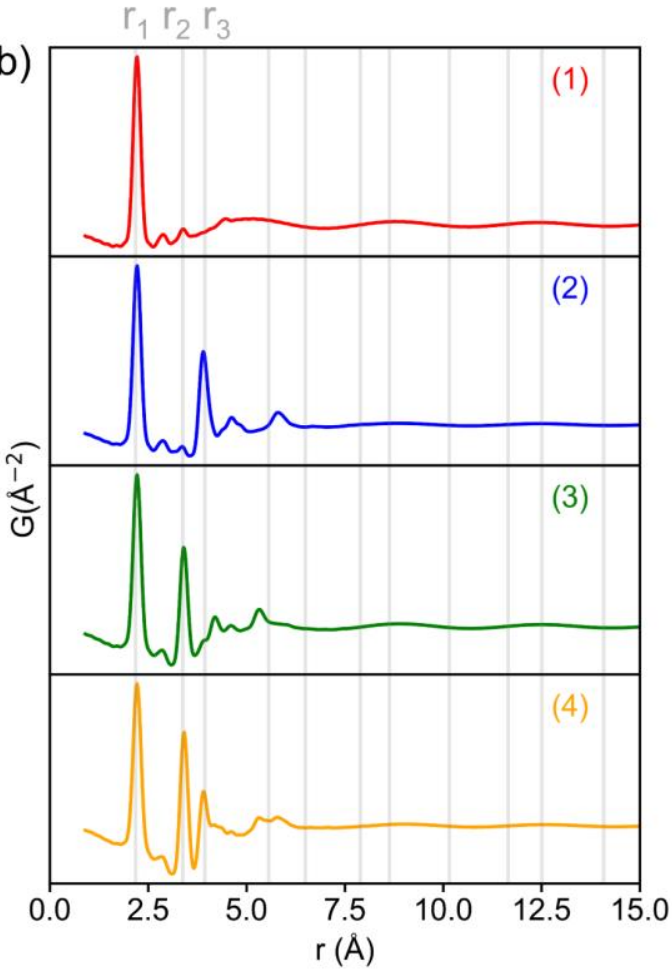

(c)

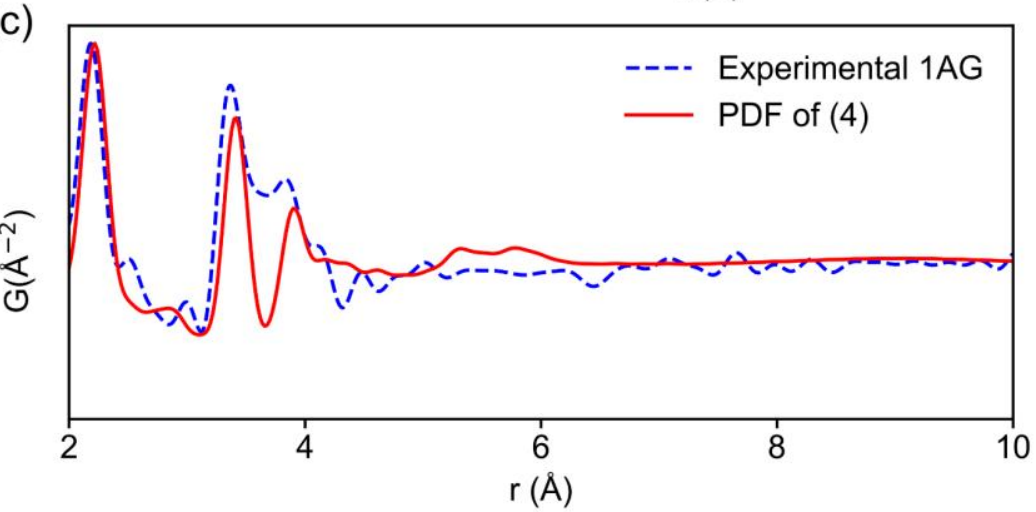

Figure S12. (a) Model clusters 1-4 with octahedra coordination ( $\mathrm{H}$ atoms are not shown); (b) Calculated PDF patterns of the model clusters; (c) Comparison of calculated PDF of cluster 4 with that of $1 \mathrm{AG}$. 

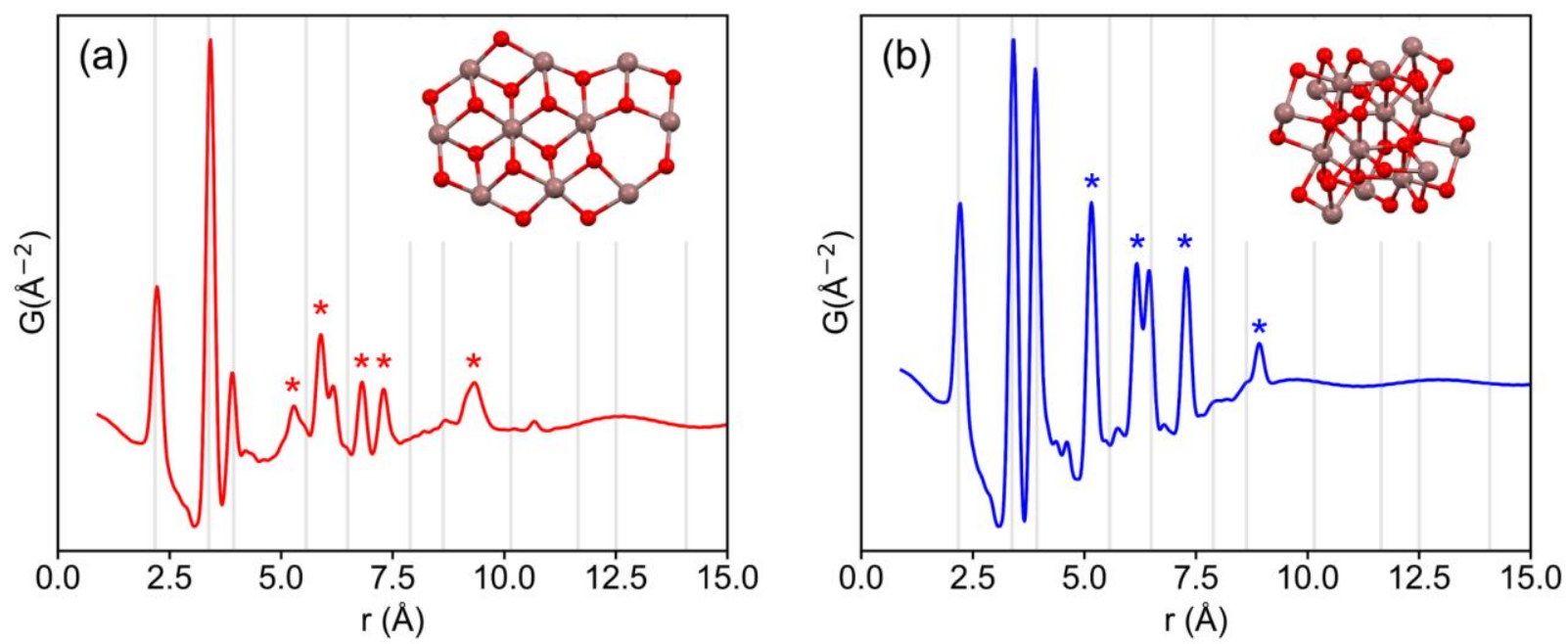

Figure S13. Calculated PDF patterns of (a) two-dimensional and (b) three-dimensional clusters extracted from the cubic $\mathrm{In}_{2} \mathrm{O}_{3}$ structure. Note: Vertical gray lines represent the peak positions of the experimental PDF of 7AG. Apparently, both two-dimensional and three-dimensional clusters shows additional atomic correlations at small-r range (marked with "*") that are not observable with the experimental PDF. In comparison, the experimental data can be best described with the linear structure shown in Figure 6.

(a)

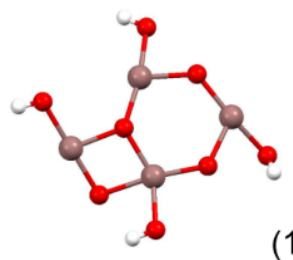

(1)

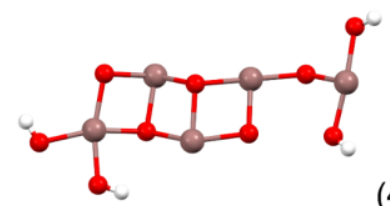

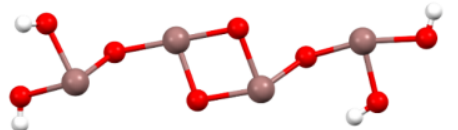

(3)

(4)

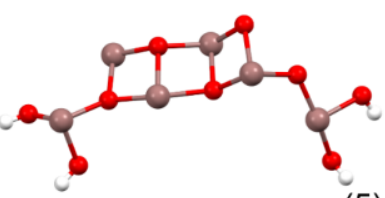

(5) (b)

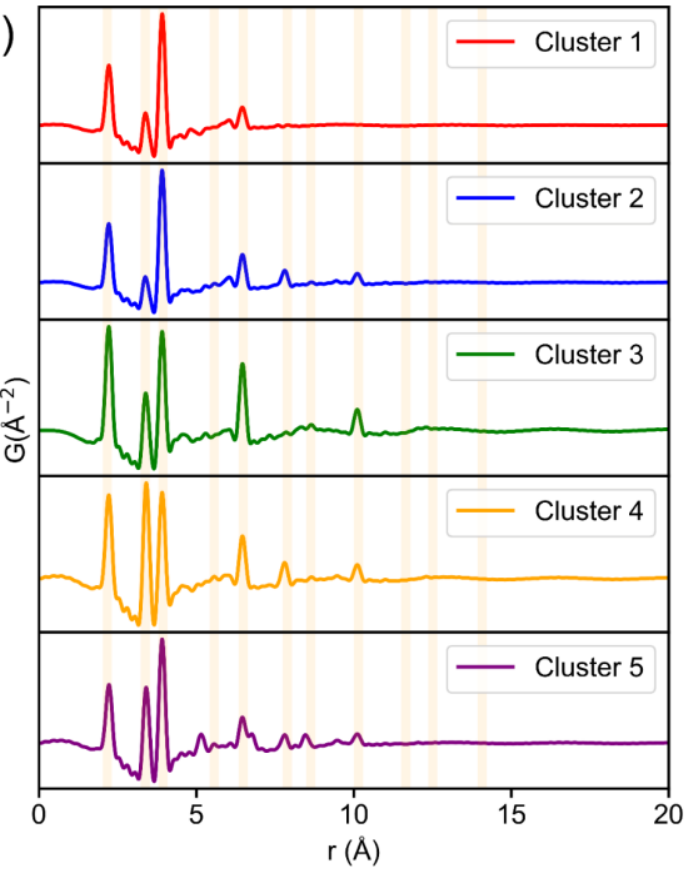

Figure S14. (a) Examples of indium-oxo clusters with variable aspect ratios and ratios of di- $\mu$ oxo-linked In and mono- $\mu$-oxo-linked In bonding patterns that could potentially exist in the asgrown samples. (b) Corresponding PDFs of the clusters exhibited in (a) (Note: The light orange lines are used to indicate the PDF peak positions for 7AG). 


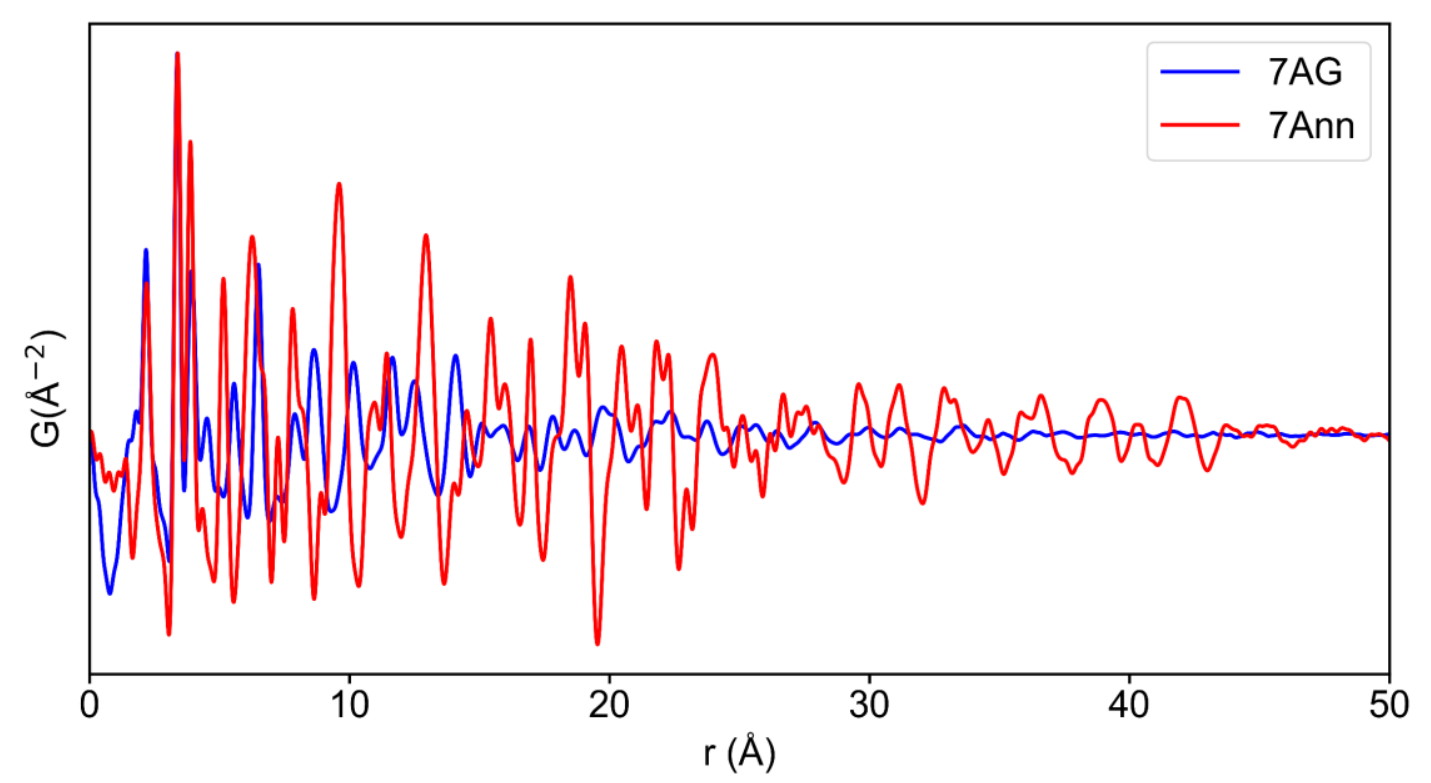

Figure S15. Comparison of PDFs between 7AG and 7Ann.

Table S1. Summary of PDF refinement values

$\begin{array}{lccccc} & \text { 1Ann } & \text { 2Ann } & \text { 3Ann } & \text { 5Ann } & \text { 7Ann } \\ \text { a } & 10.14 & 10.14 & 10.14 & 10.21 & 10.24 \\ \text { scale factor } & 0.41 & 0.68 & 0.68 & 0.97 & 2.40 \\ \text { size } & 39.67 & 41.24 & 44.57 & 49.53 & 54.22 \\ \text { delta2 } & 3.64 & 3.36 & 3.38 & 3.43 & 4.04 \\ \text { Biso(In) } & 0.68 & 0.64 & 0.65 & 1.01 & 0.96 \\ \text { Biso(O) } & 3.21 & 3.28 & 3.66 & 5.34 & 6.20\end{array}$

\section{References:}

(1) Juhás, P.; Farrow, C. L.; Yang, X.; Knox, K. R.; Billinge, S. J. Complex Modeling: A Strategy And Software Program for Combining Multiple Information Sources to Solve Ill Posed Structure And Nanostructure Inverse Problems. Acta Crystallogr., Sect. A 2015, 71, 562-568. 